\title{
Response facilitation and response inhibition produced by intracranial injections of brain extracts from trained donor goldfish*
}

\author{
WILLIAM G. BRAUD \\ University of Houston, Houston, Texas 77004
}

and

\author{
RONALD B. HOFFMAN \\ University of Houston and Texas Research Institute of Mental Science, Houston, Texas 77004
}

\begin{abstract}
Three experiments are reported which bear on the issue of whether brain extracts from trained donor animals generally facilitate learning or performance or, rather, modify recipient behavior in a specific way. In Experiment I, extracts facilitated an approach response in the absence of training (learning) of the naive recipient goldfish. In Experiments II and III, extracts from brains of inhibition-trained donor fish resulted in a significantly lower level of responding than that found in recipients of brain extracts from untrained control donor fish. Four hypotheses are described ("general learning facilitation," "generalized performance facilitation," "weak specificity," and "strong specificity" hypotheses) in which biochemical transfer results might be considered. It is shown that the present results rule out the two general-effect hypotheses and are at the same time supportive of the weak specificity hypothesis. It is shown further that, when other published findings are considered, only the strong specificity hypothesis survives as a useful description of biochemical transfer findings.
\end{abstract}

The injection of brain homogenates or extracts from trained donor animals into naive recipient animals has been reported to modify recipient behavior in a manner consistent with the training of the donors. This technique, called a "behavioral bioassay" strategy, has produced successful results in at least 33 independent laboratories throughout the world. A common criticism of this technique (e.g., Quarton, 1967) is that the brain extracts might merely enhance or facilitate learning in a general sort of way, rather than specifically modify recipient behavior even in the absence of learning. In this paper, three experiments are reported which bear directly upon this "general facilitation of learning" criticism. In the first study, a response is modified in the absence of training of recipients, and hence, the effect is upon something other than learning. In the second and third experiments, response inhibition is produced in recipients via brain extracts, eliminating the possibility of a general facilitation effect of brain extracts.

\section{EXPERIMENT I}

\section{Method}

\section{Subjects}

The Ss were 36 common goldfish, $7 \cdot 0-10.0 \mathrm{~cm}$ in body length, obtained from Ozark Fisheries, Stoutland, Missouri. Fish were maintained in groups of 11,11 (donors), 7 , and 7 (recipients) animals in 10-gal aquaria with continuously filtered and aerated water. Small fin clippings and coloration differences were used to identify individual fish. Fish were maintained in the lab for 2 weeks before the formal experiment began.

*We thank E. Brown. L. Palmer, H. and D. Ginsburg, and J. Hartnell for assistance during various stages of this research.

\section{Apparatus}

The apparatus consisted of four $30 \times 15 \times 9 \mathrm{~cm}$ plastic containers, filled to a depth of $3.75 \mathrm{~cm}$ with aquarium water. Clear white pilot lamps were attached to the ends of each container, and the containers were attached to a wooden board which could be tilted back and forth so that at any given time, one end of each container would be shallow and the other end deep. A microswitch was attached to the board so that the container ends in which the water was shallow would always be illuminated, while the other (deep) ends would be dark. The apparatus might be defined as a series of containers which contained two areas: "deep-dark" and "shallow-bright."

\section{Procedure}

Naive goldfish spent a majority of their time in the apparatus in the deep-dark end, venturing only rarely into the shallow-bright region. Approaching the shallow-bright end was chosen as the response to be trained in a group of 11 donor fish. Experimental donor fish were food-deprived for $48 \mathrm{~h}$ before training began.' Training consisted of 45 food-reinforced shallow-bright end approach trials, given at a rate of three trials every other day. A trial consisted of tilting the container and placing a food pellet (Shrimp-el-ett pelleted fish food) near the shallow-bright end; the fish was allowed $5 \mathrm{~min}$ in which to approach and feed upon the pellet. Four donor fish were run simultaneously, one in each container. A correct response consisted of an approach to the far shallow-bright end of the container and consumption of the food pellet within the 5-min interval. Eleven naive control fish were never exposed to the training apparatus and were deprived and fed in a manner identical to that of experimental donors (fed in the home tanks. of course).

Twenty hours following the last training trial, all fish were sacrificed and their brains were quickly removed and frozen in powdered dry ice. See Braud (1970) for details of the brain-removal technique. The two sets of brains (from experimental and control donors) were homogenized separately in 6 volumes of $0.85 \% \mathrm{NaCl}$ for $3 \mathrm{~min}$ in a glass homogenizing tube with motor-driven Teflon pestle. The mixtures were then stirred magnetically for $4 \mathrm{~h}$ at $4^{\circ} \mathrm{C}$. then centrifugated for $1 \mathrm{~h}$ at $40.000 \mathrm{~g}$ at $4^{\circ} \mathrm{C}$. The clear supernatants were then lyophilized. 


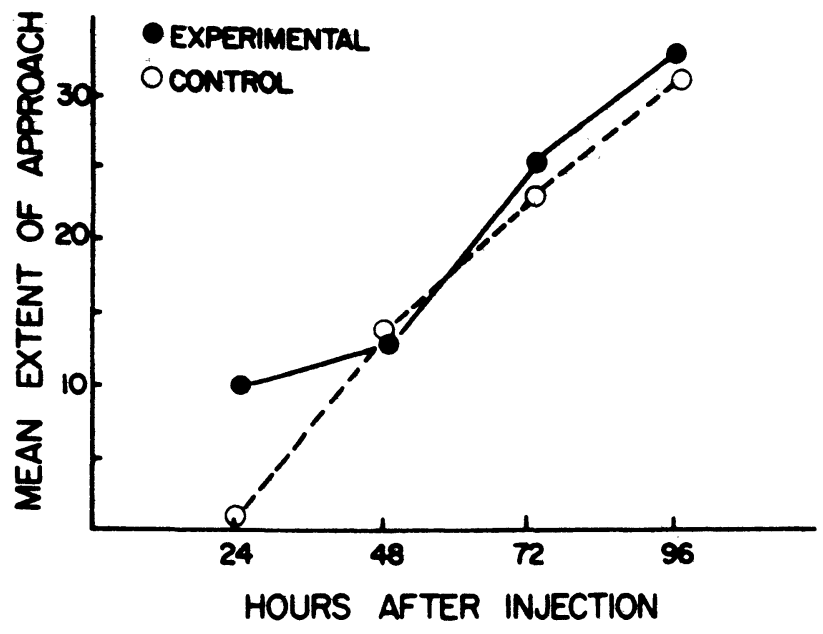

Fig. 1. Mean extent of approach to shallow-bright area in recipients of experimental (trained) and control (untrained) brain extracts as a function of hours following intracranial injection.

and the resulting dry powders were mixed with enough $10^{-3} \mathrm{M}$ $\mathrm{NaCl}$ solution to adjust the volumes of the materials so that a single $60-\mu 1$ injection (per recipient fish) would contain the equivalent of material from 1.6 donor brains.

Seven naive recipient fish were injected intracranially (see Braud, 1970) with $60 \mu \mathrm{l}$ of experimental (trained) extract; seven naive recipients were similarly injected with control (untrained) extract. Recipients were tested without reinforcement 24,48 , 72 , and $96 \mathrm{~h}$ after injection. All recipients were deprived and fed as were the control donor fish-food was never presented irr the testing environment. All recipients were given three 5-min trials per day. Approach behavior was quantified by means of the following "point system": approach within $2.5 \mathrm{~cm}$ of shallow-bright end $=4$ points; within $5.0 \mathrm{~cm}=3$; within $7.5 \mathrm{~cm}$ $=2$; within $15.0 \mathrm{~cm}=1$. An observer counted the frequency of each such approach (aided by a grid system drawn on the bottoms of the containers). Both injection and testing were done "blind" in order to eliminate $\mathrm{E}$ bias.

\section{Results}

Over the 45 training trials, donor performance increased progressively from $26 \%$ (approaches on first six trials) to $96 \%$ (approaches on last six trials). Performance of the two recipient groups is depicted in Fig. 1. Mean extent of approach is based upon all approach scores (scores of $1,2,3,4$ ) summed for a $\mathrm{S}$ and then summed and averaged across Ss. Performance is significantly superior for the experimental recipients at the 24-h test (Mann-Whitney $\mathrm{U}=10, \mathrm{p}=.036$ ). The two groups did not differ at the 48-, 72-, and 96-h test periods.

\section{EXPERIMENT II}

\section{Method}

\section{Subjects}

The $\mathrm{Ss}$ in this second study were 44 common goldfish of the same size and from the same supplier as in Experiment I. Fish were assigned randomly to two donor groups of 12 animals apiece and two recipient groups of 10 animals apiece.

\section{Apparatus}

The fish's home tanks served as the apparatus for this experiment. $l$ ish were maintained in groups of three in halves of aerated and filtered 10-gal aquaria; tanks were divided into two equal compartments by means of thin sheets of opaque brown plastic. In the center of each compartment was placed a clear plastic tube. $2.0 \mathrm{~cm}$ in diam. Into this tube (with its bottom sealed). the $\mathrm{E}$ dropped soy grit particles at the rate of one every 15 sec. Soy grits were used rather than actual food pellets because the pellets dissolved and contaminated the water. Soy grits were chosen as the food surrogate because they were of the same size and color as actual fish food and because they fell through the water with approximateiy the same veiocity as the food pellets. In preliminary pilot work, it was found that velocity was a crucial variable; fish did not strike at food surrogates which fell too slowly or too quickly.

\section{Procedure}

Fish were familiarized with the tube-containing compartments for 1 week before the experiment began. Fish were deprived for 2 days and then were fed two Shrimp-el-ett pellets per fish per day in the vicinity of the clear plastic tube. While the actual inhibition sessions were in progress, fish were fed one food pellet per day, in the vicinity of the tube, $30 \mathrm{~min}$ following the last trial of the day. Inhibition training 1 for the 12 experimental donors consisted of 50 trials per day for a period of 8 days. A trial consisted of dropping a particle of soy grits into the tube and recording whether a fish struck at the particle. Striking was defined as a rapid movement toward the falling particle, touching the tube with the snout, and following the particle down the tube. Particles fell at a rate of one every is sec (signaled by a light visible only to the E). Since the particles were nonconsumable, each of the fish had an equal opportunity to strike at them. At first, the fish struck frequently and vigorously at the particles. As inhibition progressed, strikes became less frequent and less complete.

Twenty hours following the last inhibition session, the experimental donors (as well as the 12 control donors which had not been given inhibition training, but which were deprived and fed exactly as were the experimentals) were sacrificed, their brains promptly removed and stored in dry ice, then maintained in a freezer at $-20^{\circ} \mathrm{C}$ until they were processed. An "RNA-protein" extract was prepared by the cold phenol method. The resultant of this extraction procedure is material rich in RNA but also containing appreciable protein; evidence strongly suggests that protein is the active material in these behavioral bioassays (Ungar \& Fjerdingstad, 1969). A detailed description of the physiological and biochemical phases is given by Braud (1970). The lyophilized extract was concentrated so that a single $40-\mu l$ intracranial injection would contain 1.2 brain equivalents of material.

Ten recipient fish were injected with extract from inhibition-trained donors and 10 with control extract. These naive recipients were tested at $24,48,72$, and 96 h following injection. Testing consisted of 50 soy grit presentations per session at a rate of one every $15 \mathrm{sec}$. Striking responses were recorded. Both injection and testing were again done in a "blind" fashion in order to eliminate E bias. Before injection, the two randomly constituted groups had been given a pretest consisting of 10 soy grit presentations.

\section{Results}

Examination of experimental donor performance 
revealed both a within-day and a between-days decrement in striking. The between-day decrement from over $90 \%$ strikes to close to zero strikes at the end of 8 training days demonstrates inhibition in the donor animals. Performance of recipients of naive and inhibition extracts is shown in Fig. 2. The two randomly constituted groups were virtually identical on the 10-trial pretest. At all test sessions following injection, the experimentals show an inhibited level of striking. This inhibition is statistically significant at the $24 \cdot \mathrm{h}(\mathrm{U}=$ $14.5, \mathrm{p}<.01)$ and $48-\mathrm{h}(\mathrm{U}=10, \mathrm{p}=.001)$ tests; the 72 and $96-\mathrm{h}$ tests are not significant. Thus, the recipients of extract from inhibition-trained donors showed an initially lower level of striking than did recipients of control extract.

\section{EXPERIMENT III}

\section{Method}

The third experiment was a systematic replication of the inhibition experiment just reported. Changes were introduced in (a) the number of recipient animals and (b) dosage of extract administered. All other aspects of the study were identical to those of Experiment II. Each recipient group contained $8 \mathrm{Ss}$, rather than 10 as before. Each fish received 1.5 brain equivalents of material rather than 1.2 as before.

\section{Results}

Performance of the two naive recipient groups in this replication is presented in Fig. 3. The behavioral patterns produced by the extracts are quite similar to those produced in Experiment II. Again, the randomly constituted groups did not differ on a 10-trial pretest. Again, striking response level was inhibited in the



Fig. 3. Mean percent striking responses in recipients of experimental (inhibition-trained) and control (untrained) brain extracts before injection (arrow) and as a function of hours following intracranial injection: Experiment III.

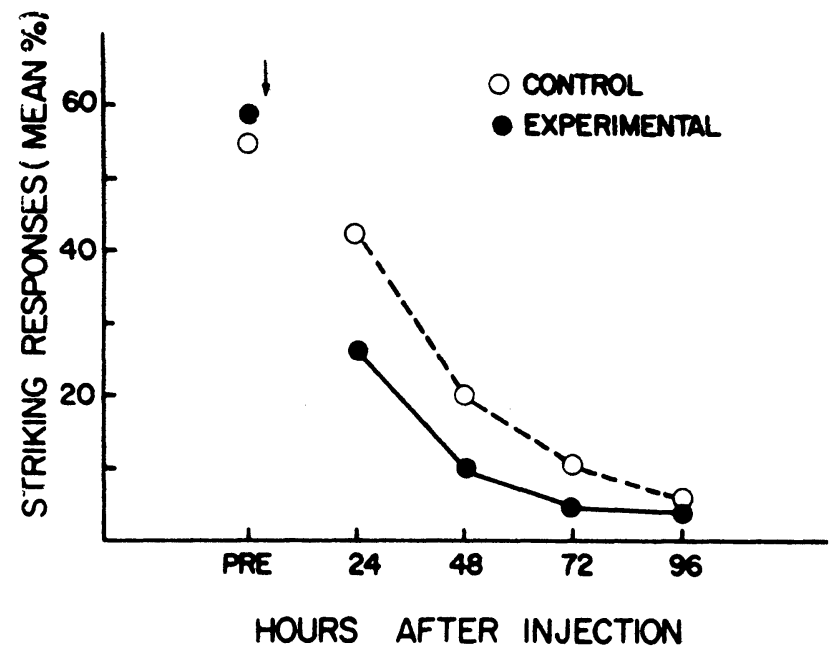

Fig. 2. Mean percent striking responses in recipients of experimental (inhibition-trained) and control (untrained) brain extracts before injection (arrow) and as a function of hours following intracranial injection: Experiment II.

experimental recipients relative to the control recipients at all 4 test days. This inhibition effect was significant at the 24-h test period $(U=12, p=.019)$, but not at the remaining three test periods.

\section{DISCUSSION}

A significant "biochemical transfer" effect occurred in Experiment I in a case involving nonreinforced testing of the recipients; thus, the effect cannot be explained in terms of a general facilitation of learning, since the recipients were not trained during testing. Experiments II and III rule out a general activating or energizing effect of brain extracts, because the significant (and replicated) effect involved a response decrement, consistent with the decremental effect occurring in the donors. These results suggest that brain extracts and homogenates do not modify recipient behavior in a very general way, but rather may result in behavior changes in recipients which mimic the behaviors occurring in the trained donor animals.

Four hypotheses might be proposed as contexts within which biochemical transfer effects might be considered. A first hypothesis (which might be termed a "general learning facilitation" notion) would suggest that brain extracts from trained donor animals might produce physiological effects which result in faster learning in recipient animals. regardless of what is learned. This sort of hypothesis would seem to be incompatible with the results of Experiment I reported herein. The hypothesis would apply only in those cases in which recipients were tested under reinforcement or learning (relearning. savings) conditions: it would not apply to positive results obtained under nonreinforced testing conditions in which recipients are not required to learn a given task. but are simply tested for the presence 
of a bias or preference (as were the Experiment I recipients). Besides those of Experiment I. results of other experiments serve to rule out the general learning facilitation hypothesis: for example. Fjerdingstad (1971) and Braud and Hoffman (1973) have obtained positive results with goldfish using nonreinforced testing paradigms. Additionally. Bryant. Santos, and Byrne (1972) have recently presented evidence contrary to the general learning facilitation hypothesis by showing that the kind of training given the recipients is of critical importance: injection of brain material facilitated the learning of a task homologous with that acquired by animals from which the brain material was isolated. but actually interfered with the learning of an antagonistic task. Thus, it is clear that brain materials do not generally facilitate or generally inhibit "learning." across the board.

A second hypothesis ("generalized performance facilitation") would suggest that brain extracts from trained donor animals generally facilitate, excite, activate, or sensitize recipients so that they are generally more active in test situations, regardless of the nature of the situation. Learning or reinforcement need not be present in such a test situation for the effect to occur. The results of Experiments II and III reported herein are incompatible with this hypothesis, since our effects were decremental or inhibitory in nature. This second hypothesis is incompatible with positive results from all studies in which "correct" behavior on the part of the recipient involves not responding either to all or to selected stimuli in its environment. Results from studies in which inhibitory processes, such as extinction (Braud, 1970) or passive avoidance (Ungar, Desiderio, \& Parr, 1972), appear to be transferred would conflict with the general facilitation of activity hypothesis. A "general inhibition of activity" hypothesis would obviously be incompatible with any incremental behavioral effects.

A third hypothesis ("weak specificity hypothesis") would suggest that some brain extracts facilitate performance or learning in some situations but not in others, while some brain extracts inhibit performance or learning in some situations but not in others. It may be the case, for example, that brain extracts from donors trained in appetitive situations facilitate recipient performance in a wide variety of appetitive situations, but not otherwise. Extracts from donors trained in aversive situations might affect recipient performance in aversive, but not in other, testing paradigms. The "weakly specific" tasks in which extracts might be active could be defined in terms of a large number of possible dimensions, for example: response inhibition tasks, tasks involving visual cues, spatial tasks, tasks influenced by certain hormones, etc. All instances within the given weakly specified class should yield similar effects; there should be no differential effects (i.e., no stronger specificity) within a given class. Our present data, taken in isolation, are supportive of this weak specificity hypothesis, as are all other data which issue from experiments in which "narrow cross-transfer" (see below:) attempts are not made. Thus, the bulk of all "transfer" experiments would fall into the class of studies which support the weak specificity hypothesis as opposed to the two general-effect hypotheses mentioned above. Rarely, however, are both incremental and decremental effects reported in the same study, as they are in this report.

A final hypothesis ("strong specificity hypothesis") would suggest that rather specific information is somehow conveyed or initiated by donor brain material. Such information would be specific enough to be used by recipients only within a very narrow range of testing situations. On this view, different effects could obtain within a class of situations (such as appetitive or aversive or light-signaled situations, etc.), with recipient behavior mimicing donor behavior only. under the precise conditions under which the donors were trained (with some leeway allowed for generalization gradient effects, and so on). Support of this strong specificity hypothesis has been the goal of those doing behavioral research on the biochemical transfer problem. Evidence very highly supportive of the strong specificity hypothesis include the following: (a) habituation to sound produces a decrement to sound, and habituation to airpuff produces a decrement to airpuff in recipients, but not vice versa (Ungar, 1967); (b) whether recipients approach a click or a light depends upon the particular stimulus used in training the donors (Jacobson. Babich, Bubash, \& Jacobson, 1965); (c) Domagk and Zippel (1971) have shown intramodality stimulus specificity effects using different colors and different tastes and odors in biochemical transfer studies with goldfish; (d) Braud and Hoffman (1973) have demonstrated intramodality specificity using upright vs inverted triangular shapes as specific cues for goldfish in transfer studies; (e) Ungar, Galvan, and Chapouthier (1972) have shown recipient effects to be specific to the particular color stimuli used in training donor goldfish; (f) extracts from donors trained in one task (dark avoidance vs step-down avoidance) modify recipient behavior in the same task but not in the other task (Ungar, 1970; Guttman \& Gronke, 1971); (g) extracts from learners in an avoidance task produce a significant transfer effect; while extracts from nonlearners exposed to the very same stimuli do not (Braud, 1971); (h) acquisition extract facilitates acquisition but not extinction, while extinction extract facilitates extinction but not acquisition (Braud, 1971); and (i) specific effects occur in circle-size discrimination tasks in hooded rats (Braud \& Braud, 1972). In all of these studies, certain brain extracts facilitated performance in certain specific situations, but had no effect in others. In "narrow cross-transfer" designs (e.g., Braud \& Hoffman, 1973; Ungar et al 1972), the situations in which extracts had no effect were very similar to those in which they were effective (e.g., upright vs inverted triangular shaped stimuli; blue vs green stimuli). Other published and 
unpublished findings favor the same strong specificity hypothesis. Further studies should be done in which the limits of the specificity effect are explored. Such studies are now in progress in our lab as well as in those of other researchers.

\section{REFERENCES}

Braud, W. G. Extinction in goldfish: Facilitation by intracranial injection of "RNA" from brains of extinguished donors. Science, 1970, 168, 1234-1236.

Braud, W. G. Modification of recipient behavior by intracranial injections of extracts from brains of trained donor goldfish. Paper presented at the First Annual Meeting of the Society for Neuroscience, October 1971, Washington, D.C.

Braud, L. W., \& Braud, W. G. "Biochemical transfer" of relational responding (transposition). Science, 1972, 176, 942-944.

Braud. W. G., \& Hoffman, R. B. Response-, stimulus-, and process-specificity in behavioral bioassays. Journal of Comparative \& Physiological Psychology, 1973, in press.

Bryant, R. C., Santos, N. N., \& Byrne, W. L. Synthetic scotophobin in goldfish: Specificity and effect on learning. Science, 1972, 177, 635-636.

Domack, G. F., Zippel, H. P. Chemical transfer of learned information in goldfish. In E. J. Fjerdingstad (Ed.), Chemical transfer of learned information. New York: American Elsevier, 1971.

Fjerdingstad, E. J. The goldfish as an experimental subject in chemical transfer. In E. J. Fjerdingstad (Ed.), Chemical transfer of learned information. New York: American Elsevier, 1971.

Guttman, H. N.. \& Gronke, L. Passive transfer of learned dark and step-down avoidance. Psyshonomic Science. 1971, 24, 107-109.
Jacobson, A. L., Babich, F. R., Bubash, S., \& Jacobson, A. Differential approach tendencies produced by injection of RNA from trained rats. Science, 1965, 158, 636-637.

Quarton, G. The enhancement of learning by drugs and the transfer of learning by macromolecules. In G. Quarton, T. Melnechuk, and F. D. Schmitt (Eds.), The neurosciences. New York: Rockefeller University Press, 1967.

Ungar, G. Transfer of learned behavior by brain extracts. Journal of Biological Psychology, 1967, 9, 12-27.

Ungar, G. (Ed.). Molecular mechanisms in memory and learning. New York: Plenum, 1970.

Ungar, G., Desiderio, D. M., \& Parr, W. Isolation, identification and synthesis of a specific-behaviour-inducing brain peptide. Nature, 1972, 238, 198-202.

Ungar, G., \& Fjerdingstad, E. J. RNA-bound peptides in chemical transfer of learned behavior. Molecular Neurobiology Bulletin, 1969, 2, 9.

Ungar, G., Galvan, L., \& Chapouthier, G. Possible chemical coding for color discrimination in goldfish brain. Experientia, 1972, 28, 1026.

\section{NOTE}

1. Throughout this paper, we have used the more neutral term "inhibition" to refer to this operation and its results. The observed decrease in strength of the striking response might qualify as "habituation," "experimental extinction," or "passive avoidance," depending upon one's theoretical orientation. Since we have not determined all the necessary defining characteristics of the effect, we prefer to call it simply "inhibition."

(Received for publication May 23, 1972; revision received December 15,1972 .) 